\title{
Women's Perspective on Using Tinder
}

\author{
A User Study of Gender Dynamics in a Mobile Device Application
}

\author{
Milena Ribeiro Lopes \\ Trinity College Dublin \\ lopesm@tcd.ie
}

\author{
Carl Vogel \\ Trinity College Dublin \\ vogel@tcd.ie
}

\begin{abstract}
Mobile applications expand possibilities for interaction and extend the boundaries of communication. Regarding online dating, Tinder is one of those applications that make it easier to connect people, and it brings out a new concept of relationship in which emotional bonds are established in a virtual space. However, it is relevant to investigate whether Tinder enables women's empowerment - how the user-mobile device interaction and the graphical user interface bear upon gender dynamics. Forty women were interviewed and the findings show that Tinder is not meeting women's expectations, rather it is stimulating the objectification of women. Considering that online dating is a growing trend and that it can be a revolutionary tool to connect people, we analysed the interface and pointed out some issues that should be considered to enhance women's experience.
\end{abstract}

\section{CCS CONCEPTS}

- Human-centered computing $\rightarrow$ User studies; Graphical user interfaces; User interface design;

\section{KEYWORDS}

User-Mobile Device Interaction; Gender Dynamics; Online Dating Application

ACM Reference format:

Milena Ribeiro Lopes and Carl Vogel. 2017. Women's Perspective on Using Tinder. In Proceedings of SIGDOC Conference, Halifax, Canada, August 2017 (SIGDOC 2017), 11 pages.

https://doi.org/

\section{INTRODUCTION}

Cyberspace can be considered a safe environment in which to engage and deepen intimacy, due to its support of virtual connections [18]. Despite this apparent safety, some concerns arise exactly from the ease of interaction. Since Tinder encourages relationships, first inside a virtual space and later, potentially, with physical interaction, it is relevant to understand the interaction in relation to gender issues and sexist patterns already established by society.

This study aims to analyse Tinder as a social influence upon gender dynamics and to understand how women feel during their experience with the application. For that purpose, we describe our analysis of interviews about Tinder with 40 women. The idea of

Permission to make digital or hard copies of part or all of this work for personal or classroom use is granted without fee provided that copies are not made or distributed for profit or commercial advantage and that copies bear this notice and the full citation on the first page. Copyrights for third-party components of this work must be honored.

For all other uses, contact the owner/author(s).

SIGDOC 2017, August 2017, Halifax, Canada

(C) 2017 Copyright held by the owner/author(s).

ACM ISBN

https://doi.org/ the experiment came after informal talks with female users. They would frequently reveal contradictory feelings about the application as excitement and frustration. From this first impression, a question emerged: "Is Tinder meeting women's expectations and needs?" Research in the field of human-mobile device interaction brought awareness of social patterns as factors that should be reasoned and planned during the development of interaction and the Graphical User Interface (GUI) which supports the interaction.

Currently, many cultures are embracing the concept of multiple genders in opposition to the traditional idea of gender binarism in which people are simply defined as male or female. Although the present research brings out some sexist concerns that often fall into the male-female polarity, it is not at odds with a more complete gender spectrum or with relationship diversity. To evaluate how women feel in relation to the application, sexual preference was not taken into account. However, we hypothesize that male-female interaction comprises historical patterns of behavior that can be reproduced in new technological developments and can negatively affect women's empowerment and gender equality. Further, we also hypothesize that sexist behaviors can be incited by the user-mobile device interaction, and due to this, interfaces should be carefully planned and designed. This research focuses in the female point of view of the interaction with Tinder. The investigation on how male perceive the application could lead to different results. However, this does not change how women currently feel about it, and it does not change their experiences.

Behavior patterns within the virtual realm may differ from in embodied interactions: sometimes gender inequality can be intensified because people feel more confident to express their views at the distance supplied by virtual environments, but this can be mitigated through the fast spread of equalitarian thoughts by media campaigns and by social media influencers. In relation to this freedom, it is necessary to understand what freedom encompasses in gender relations since the virtual communities reproduce established social patterns and power dynamics [18]. Thus, the structures of a patriarchal society will be reproduced among its media channels and through technological developments including mobile applications. One example of the anti-feminism movement is the rise of online communities such as "Men's Rights Movement" (MRE), "Men Going Their Own Way" (MGTOW), the "Red Pill", among others, which oppose the ideal of gender equality [13]. For this reason, it is necessary to check whether Tinder, as a communication vector, is spreading gender equality or worsening inequality.

"Harassment" is arguably an over-used term and is correspondingly problematic as a conceptual notion. A recent study about sexual harassment [2] tries to differentiate solicitation from derogation and arguess that sometimes what victims perceive as sexual harassment is actually a solicitation act targeting short-term sexual encounters. 
However, because the purpose is to understand how women feel, users' opinions about feeling offended or not is what defines the use of the term in the present research.

On one hand we can say that women within western culture have gained more independence within relationships and more control over relationships due to gains such as reproductive rights, family law, equal employment rights, property rights and so on. On the other hand sexist behavior and all sort of gender violence still pervade virtual and physical interactions. According to Wajcman [18], technology is "an extension of patriarchal and capitalist domination" and "has been shaped by men to exclusion of women." From her perspective, there is a tendency to reinforce gender hierarchies within technological developments. Considering this scenario where women feel free to benefit from technological advances in a world still influenced by gender hierarchies, how should an application provide healthy and easy interactions and at the same time provide empowerment, safety, self-confidence and respect to women? The first step is to understand how they feel in relation to that kind of interaction, that goes far beyond the virtual bonds and incite the interaction in its deepest level of intimacy.

For that purpose, we established three research goals: to learn whether the application meets women's expectations, to investigate whether there are sexist patterns of behavior during the Tinder experience and to check which elements of the interface contribute to a good or bad experience regarding gender dynamics. This research is independent of Tinder - it is conducted without support from Tinder nor any link or agreement with the company.

\section{A BRIEF TINDER OVERVIEW}

Tinder is an online dating application which is used worldwide. It is increasingly popular, and it is changing the way people engage. Based on Tinder's last report [1] the application is been used in 196 countries and makes possible 26 million matches a day. According to the company the application is focused on bringing people together and promoting connections that would not be possible outside the virtual realm. Indeed, the online dating applications is somehow revolutionary in terms of the connectivity it enables.

Tinder's graphical user interface is quite simple and clear, and that contributes to the popularity of the application. It shows one person at a time and to see the next person the user must take a decision: to like or dislike the person viewed. The communication through Tinder has only two steps. The first step is choosing possible partners/friends. The idea of the manipulation is to be as simple as possible, and interactions require essentially three gestures (see figure 1): the swipe left (dislike), the swipe right (like) and the swipe upwards (super like). The "super like" feature is limited in availability per day. When the user super likes someone it will make the interest explicit, so the other part will know that the user is interested before he/she takes an action (swiping right or left). That is the main part of the interaction: choosing people. Once one likes a person one needs to wait to see if that person reciprocates within the next hours or days (it depends on how often the other user goes online). Otherwise the other person will not know that one has liked $\mathrm{him} / \mathrm{her}$, because feedback is provided when there is a mutual match (except for superlikes). Not matching can mean that the person does not reciprocate, but not necessarily. It can happen that the person did not see the profile because he/she is not online often or does not spend much time swiping. The second and last step of the interaction is chatting. People may maintain some active conversations with their matches through the application's chat system.

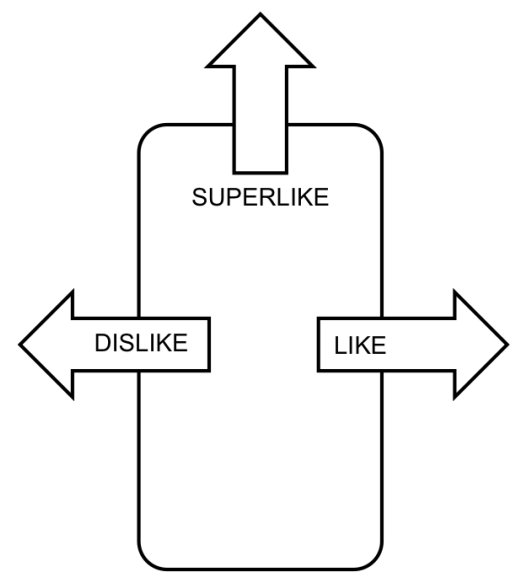

Figure 1: Tinder's basic set of gestures to interact with the GUI.

Evident appeal of the system interface derives in part from the reduction of personal risk in expressing unreciprocated attraction to an image of another person. However, this comes with a privacy cost since the interface gestures required may be visible in public, known to those acquainted with the application, and therefore potentially at odds with the deepest wishes of an application user who might rather certain others not to know that they use the application. The potential gain from consequent positive interactions enabled by the application appears to outweigh the risks for many users.

\section{RESEARCH METHOD}

The research has a mixed methods approach characterized by the incorporation of both qualitative and quantitative data [8]. The approach would be considered as qualitative regarding the subject and purpose of the study; however, we decided to integrate the quantitative approach to the analysis of the findings, which would help to establish facts about the distribution of perspectives on sensitive matters (female perception of an online dating application).

\subsection{Data generation}

The research method involves data generation [4] through the use of a structured interview with open-ended questions, text analysis of responses, the transformation of the interview information into statistical data and the analysis of the results. Although an unstructured interview might be recommended due to the qualitative nature of the research questions [7] we considered the structured interview the best option to focus on the subject and goals. The open-ended response approach enables to understand the different points of view around each question [14]. The interview guide was composed of fourteen items but some of them contain more than one question per item. The interview was planned to be as neutral as possible. The questions were organized following the natural stages of conversation in the way that the interviewees could find easier to remember 
their experiences with the application and describe those experiences. The questions and the order were planned in a way to obtain information about participants' expectations, impressions and feelings about gender dynamics.

The interview method and the structured interview guide were submitted to the Research Ethics Committee of the School of Anonymous of Anonymous, before any materials were provided to interviewees, and the research was approved by this research ethics committee. Participants initially received an information sheet describing the study, and then a consent form. Upon receipt of a completed consent form, participants were sent the questions by email.

\subsection{Participants and recruitment}

The sample is qualified as a nonprobability convenience sample composed of forty Brazilian women who have used Tinder at least once to meet new people through the Internet. The recruitment was conducted through Facebook. The first call for volunteering was posted in a Facebook closed group of Brazilian females living in Ireland. The group had 8,074 members at the time of recruitment (April, 2016). The call was posted only one time in this group. The invitation described the research topic and what would be involved in participating. Women interested in participating in the survey were asked to leave a comment or an inbox message with their email address. 111 women expressed interest. Another call was posted to friends in the personal Facebook page of one of the authors eliciting expressions of interest from 14 more women. Some of theses 125 original women invited participation from another nine women, whose email addresses were given with the permission of those nine. In total 134 women expressed interest in participating. The consent form approved by the university ethics committee was sent to them. However, not all responded with consent to the research terms and conditions, and even fewer responded to the interview subsequent to providing informed consent. From the 134 total women that gave their emails only 40 were interviewed. This high drop-out rate could be attributed partly to the length of the information sheet and consent form that is necessary to make sure that all the ethics issues are covered. Attrition may also be partly attributed to the lack of immediacy in conducting interviews by email. Despite this, the interviewees are easily reached by email and email participants have ample time to reflect and to reply at their own convenience [12]. We believe that, in general, the benefit of using the email to administer the interviews compensates for the losses.

Although our findings cannot be extended to the whole population of women who use Tinder, analysis reveals significant results that give a picture of the gender dynamics on Tinder. Age and education level were not taken into consideration in the selection of participants (we did not ask participants to provide such information about themselves). However, for one comparison between questions we had to consider their sexual preference in order to assess consistency of response. From the 40 women interviewed only one claimed to be looking for homosexual interaction (in the case, exclusively for a relationship). Nationality is a variable that affect the research and our idea is to have a sample with mixed nationalities in future studies. However, due to the opportunity sampling (target population available and willing to take part in it) we used the population of Brazilian women.

\subsection{Procedures}

The interviews were self-reported by email and returned by the interviewers within a month. We reminded participants about the motivation of the research (to understand how women feel during their experience on Tinder). We provided, in Brazilian Portuguese, all the questions to each participant (see Appendix), as a way of opening communication for further discussion. There were no word count limits on their answers (open-ended) and they were also free to skip questions and to respond with only what they felt comfortable. The interview was planned to be answered by email since we believed women would feel more confident to talk about their experiences through writing. We also believed they would have more time to think and remember their experiences before answering, since mediating interviews via email enables participants to respond at their leisure, with all of the opportunity for reflection that they might wish [12]. Conversely, the lack of a face-to-face communication also entails reduced interactivity and correspondingly reduced chances of richer answers from interviewees. However, as the answers could be highly affected by a direct dialogue, it was finally decided that for the purpose of this research the better option for obtaining a neutral perspective about their experiences would be a structured interview by email. A response deadline was not imposed; however, only responses received before 11-May-2016 were analyzed.

\subsection{Data analysis}

The original fourteen questions were split into seventeen questions (since some of them had more than one question inside each question) for analysis purposes (see Appendix). Question 12 was not considered in the quantitative analysis, and responses to it are not included in the findings, as the question did not elicit quantifiable response. Data analysis was divided into three steps. First, answers were carefully analysed with manual close reading, and it was possible to categorize the standard answers and to classify each answer according to those standards. Secondly, descriptive statistics were generated with R. Finally, the statistics were analysed to find correlations between the answers and categories of answers that helped to bring to light an overview on how the women feel about Tinder.

As the data was generated from the interview responses, for many questions, the interviewees gave more than one answer. This was permitted as the intention was to let them express as much as they wished. Thus, the tables below report for each question the number and percentage of respondents who included the relevant answer category within their answer; and because of multiple categories of reply, in some answers the sum of options of answers is not $100 \%$. The relevance of the statistics bear on the proportion between the answers. We want to find out how women feel, and for that reason the value of the results of each question bear on the rates, frequency and the comparison with other questions' responses.

\section{INTERVIEW FINDINGS}

Question 1 of the interview was about the purpose for installing the application (see table 1 below). $60 \%$ have pointed out that they were looking for friends or meeting new people, among other interests; $40 \%$ were looking for relationship among other interests; $7.5 \%$ were looking for casual sex among other interests. Only $22.5 \%$ were exclusively interested in a relationship, and none of the 40 women 
was exclusively interested in sex. Because many women are living abroad (in Ireland), 22.5\% reported using the application for practising language (English). In order to analyse if the application is meeting user expectations, it is helpful to understand user intentions in adopting the application.

\begin{tabular}{lrr}
\hline Motivation to use Tinder & No. of Women & $\%$ \\
\hline Find friends & 24 & $60 \%$ \\
Find a relationship & 16 & $40 \%$ \\
Practice language & 9 & $22.5 \%$ \\
Curiosity & 4 & $10 \%$ \\
Find casual sex partners & 3 & $7.5 \%$ \\
Other reasons & 1 & $2.5 \%$ \\
\hline
\end{tabular}

Table 1: Women's motivations on Tinder: each woman could have pointed out more than one motivation and the sum is not $100 \%$. 40 women responded to this question; therefore none of them was absent.

Question 2 is about reflections prior to installing the Tinder application. The idea was to check whether there was any concern or preconceived idea about the application. From the total respondents, $64.1 \%$ did not report having internal debate before installing it. Those who reflected at greater length mostly said this was due to one of these reasons: they were afraid of being recognized and judged; they did not like the sexual approach of this kind of application; they did not like virtual approach and prefered to find people in real life; they did not want to cause pain to ex-partners in the case they would find out. The responses to this question (see table 2) shows that the majority felt comfortable about using an online dating application. Although some were still concerned about using it (35.9\%) the benefits of an online dating application surpassed the concerns. However, it is possible to assume that some number of women gave up using the application for the same reasons those interviewees have pointed out in response to this question.

\begin{tabular}{lrr}
\hline Any concern prior to installation? & No. of Women & $\%$ \\
\hline No & 25 & $64.10 \%$ \\
Yes & 14 & $35.90 \%$ \\
\hline Total respondents & 39 & $100 \%$ \\
\hline
\end{tabular}

Table 2: About pondering before install Tinder: the respondents gave only one answer, therefore the sum is $100 \%$. 39 women responded to this question and one provided no response.

Question 3 asked participants about their first impression using the application. Considering only those who answered the question (38), the most frequently expressed response (26.3\% of respondents) is that the system is superficial (see table 3 ). Considering all the 38 responses to this item and dividing it into positive and negative feelings it is possible to say that $52.6 \%$ had a first bad impression (since the unfiltered refers to the lack of filters and sexualized refers to being too geared toward sex), while $47.4 \%$ had a good first impression. For this question, we have considered one response for each interviewee - this was possible because they did not provide ambiguous responses with respect to positive and negative categories (as, for exemple, "confident" and "sexualized"); those who mentioned more than one feeling for the first impression concentrated it into the "negative" or "positive" feedback.

\begin{tabular}{llrr}
\hline Category & First impression & No. of Women & $\%$ \\
\hline Negative & Superficial & 10 & $26.3 \%$ \\
Positive & Exciting & 9 & $23.7 \%$ \\
Negative & Unfiltered & 7 & $18.4 \%$ \\
Positive & Fun & 7 & $18.4 \%$ \\
Positive & Easy & 4 & $10.5 \%$ \\
Negative & Unsafe & 3 & $7.9 \%$ \\
Negative & Sexualized & 1 & $2.6 \%$ \\
Negative & Invasive & 1 & $2.6 \%$ \\
\hline
\end{tabular}

Table 3: Women's first impression about Tinder. Respondents could point out more than one impression, therefore the sum is not $100 \%$. 38 women responded to this question and two provided no response.

\begin{tabular}{lrr}
\hline First impression: positive or negative? & No. of Women & $\%$ \\
\hline Total Negative & 20 & $52.6 \%$ \\
Total Positive & 18 & $47.4 \%$ \\
\hline Total respondents & 38 & $100 \%$
\end{tabular}

Table 4: Overall impression based on the positive-negative polarity. None of them pointed out both negative and positive feelings.

Question 3 also asked participants how they felt about their first matches (see table 5). In relation to this, $84.4 \%$ of those who answered the question expressed feeling confident, $15.6 \%$ had a neutral reaction and no one expressed any negative feeling. Despite the results of last the question, where $52.6 \%$ reported a bad first impression of the system, they would mostly feel good about matching someone, and could have compensated for negative first impressions to some extent, maintaining their participation the Tinder system for longer.

\begin{tabular}{lrr}
\hline Feeling after matching someone & No. of Women & $\%$ \\
\hline Confident & 27 & $84.4 \%$ \\
Neutral & 5 & $15.6 \%$ \\
\hline Total respondents & 32 & $100 \%$
\end{tabular}

Table 5: How women felt after their first matches. Each women pointed out just one feeling. 32 women responded to this question and eight were absent.

Regarding the application interaction (question 4), the match mechanism and the way it enhances the interaction, 18 women gave positive feedback saying that it enhances privacy (since one only learns of ratings of another if the ratings are of mutual interest) or reciprocity (diminishing the risk of feeling rejected) or is assertive, innovative or empowering. Eleven women gave negative feedback and said the experience was superficial. Four indicated that the system was superficial but also innovative, assertive or that enhances privacy. Two said they had a neutral feeling about it. Thus, from 
the 35 women who answered the question, $51.43 \%$ gave positive feedback, $31.43 \%$, negative and $17.14 \%$, mixed or neutral feedback. The result of all the feedback combined is in table 6 . This question reveals the potential and weaknesses of the application. From this question it is possible to perceive that, on one hand, the match system enhances confidence through privacy and reciprocity; however, on the other hand, it increases the "disposability of people" through a system based on binary classification and on appearances alone, as they have more fully explained in the interviews.

\begin{tabular}{llrr}
\hline Category & Match system & No. of Women & $\%$ \\
\hline Negative & Superficiality & 15 & $42.9 \%$ \\
Positive & Privacy & 11 & $31.4 \%$ \\
Positive & Reciprocity & 5 & $14.3 \%$ \\
Positive & Assertive & 4 & $11.4 \%$ \\
Positive & Innovative & 3 & $8.6 \%$ \\
Neutral & Neutral & 2 & $5.7 \%$ \\
Positive & Empowering & 1 & $2.9 \%$ \\
\hline
\end{tabular}

Table 6: User's impressions about the match system: They could point out more than one impression, therefore the sum is not $100 \% .35$ women responded to the question and five were absent.

Question 5 probed positive aspects of experiences with Tinder (see table ??). 65\% of those who answered met nice people and/or made good friends (meeting with the expectation of the $60 \%$ who have claimed to be interested in friendship in response to question 1). Long or short-term relationships were found by $47.5 \%$, even though only $40 \%$ expressed interest in establishing relationships through the system originally (see table 1). $17.5 \%$ indicate having experienced no benefit from using the application. These findings demonstrate the efficacy of the application both for meeting new people and for finding partners, the most frequently expressed interests.

Question 6 probed negative aspects of the application (see table 7). Unpleasant experiences of a direct sexual approach were reported by $50 \% ; 15 \%$ expressed being insulted by the users and $15 \%$ experienced a sexist behavior. A small but startling number (two users) reported having been reprimanded by male friends who saw their profiles on Tinder and felt free to say that it was not a place for them and that the women should not be using it. Both were living in their hometowns when it happened. For the majority recently living abroad that kind of behavior is less likely since it is then harder to see known people on the application. The direct sexual approach is offensive in many ways. That is probably the worst aspect of the application to emerge in this study, since the users seem to feel free to drop the social norms of behavior of face-to-face conversations, revealing sexist thought that is hidden by societal rules. ${ }^{1}$ In total, 28 women related an offensive experience regarding gender dynamics (see table 8 ). This represents $70 \%$ of all interviewees.

From the answers about feeling respected or not during their experience (question 7; see table 9), it was possible to find a spectrum that would range from "not at all" to "Yes, completely" including the "yes and no" and the "mostly yes" or "mostly no" in the middle. The

${ }^{1}$ It is also a possibility that rather than revealing hidden thoughts, the norms of Tinder actively encourage kinds of thought that would not normally be entertained during direct interaction.

\begin{tabular}{lrr}
\hline Negative side of the experience & No. of Women & $\%$ \\
\hline I felt harassed by direct sexual approach & 20 & $51.3 \%$ \\
I was insulted & 6 & $15.4 \%$ \\
I was a victim of sexist behavior & 6 & $15.4 \%$ \\
The application provides low interaction & 5 & 12.8 \\
The application lacks filter & 3 & $7.7 \%$ \\
There is no negative side & 3 & $7.7 \%$ \\
The application is superficial & 3 & $7.7 \%$ \\
Other & 3 & $7.7 \%$ \\
I was a victim of racist behavior & 2 & $5.1 \%$ \\
I was reprimanded for using Tinder & 2 & $5.1 \%$ \\
The application is addictive & 1 & $2.6 \%$ \\
\hline
\end{tabular}

Table 7: Negative side of the experience with Tinder: each woman could have pointed out more than one motivation and the sum is not $100 \% .39$ women responded to this question and one was absent.

\begin{tabular}{lrr}
\hline Report of offensive behavior & No. of Women & $\%$ \\
\hline Reported offensive behavior & 28 & $71.8 \%$ \\
Did not reported offensive behavior & 11 & $28.2 \%$ \\
\hline Total respondents & 39 & $100 \%$ \\
\hline
\end{tabular}

Table 8: Ratio of women who experienced at least one of these offensive behaviors: felt harassed by direct sexual approach, was insulted, was reprimanded, was a victim of sexism or was a victim of racism.

results show that there is a very balanced amount of women between positive and negative poles, but the majority falls in the middle where they would relate their experience as a mix of respectful and disrespectful moments.

\begin{tabular}{lrr}
\hline Did you feel respected? & No. of Women & $\%$ \\
\hline Yes & 9 & $22.5 \%$ \\
Mostly yes & 5 & $12.5 \%$ \\
Yes and no & 12 & $30.0 \%$ \\
Mostly no & 3 & $7.5 \%$ \\
No & 11 & $27.5 \%$ \\
\hline Total respondents & 40 & $100 \%$ \\
\hline
\end{tabular}

Table 9: Feeling of respect during the experience on Tinder.

Comparing the response pattern for question 7 with the results of question 6 , we found out that among those 28 women who reported being offended, experiencing a direct sexual approach, encountering sexist or racist behavior or reprimand from others for using Tinder, five still expressed feeling fully respected (see table 10. This fact may suggest that some women grew used to that kind of behavior and, despite disliking it (since they have pointed it out as a negative side of their experience) they do not feel disrespected, otherwise they would not feel fully respected.

The application's engagement was measured by the time of use (see table 11), and reason to uninstall (see table 12) (questions 8 and 9). The $48.65 \%$ of the 37 participants who answered the question had used the application for less than six months; however $32.43 \%$ had 


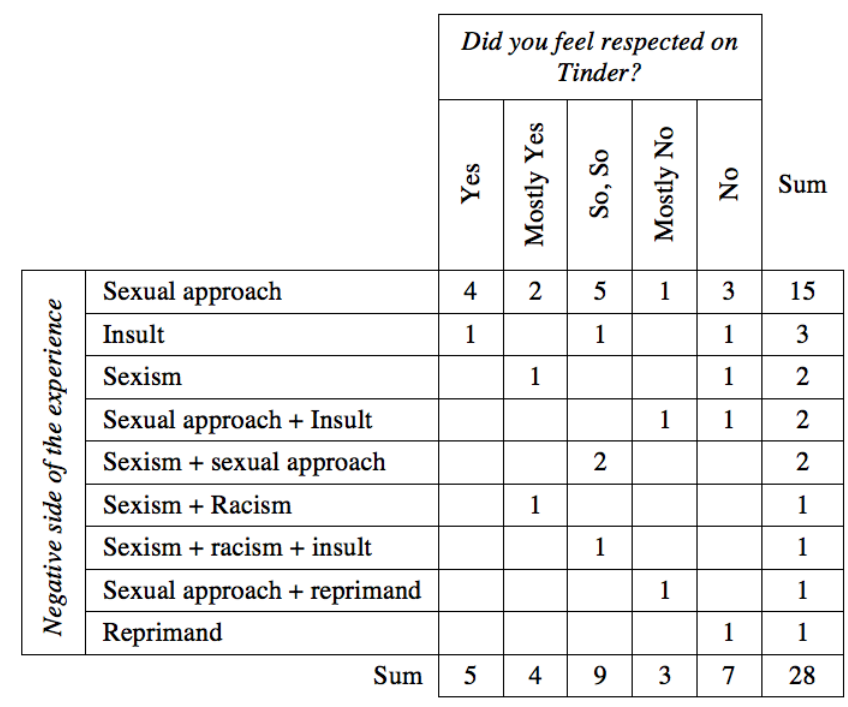

Table 10: Comparison by the feeling of respect during the experience on Tinder and the report of offensive behavior among the 28 women who reported one or more types of offensive behavior.

used for more than a year. From the original sample, 35 women had uninstalled the application and $42.86 \%$ from a sample of 35 provided their reason to uninstall pointed out relationship, another $42.36 \%$ pointed out frustration, $14.3 \%$ had lost their interest in the application and $5.7 \%$ said they had uninstalled because they were reprimanded by known people for using Tinder. From the 35 women, 20 have uninstalled the application due to a negative experience (frustration, lack of interest and repression), two due a mix of negative experience and the start of a relationship and 13 have quit the application only because they started a relationship. Hence, the rate of $57.1 \%$ that uninstalled it exclusively for a negative experience represent the majority of the interviewees.

\begin{tabular}{lrr}
\hline For how long did you use Tinder? & No. of Women & $\%$ \\
\hline up to 6 months & 18 & $48.65 \%$ \\
up to 1 year & 7 & $18.92 \%$ \\
more than 1 year & 12 & $32.43 \%$ \\
\hline Total respondents & 37 & $100 \%$ \\
\hline
\end{tabular}

Table 11: Time of use of the application.

\begin{tabular}{lrr}
\hline Why did you uninstall the app? & No. of Women & $\%$ \\
\hline I've started a relationship & 15 & $42.86 \%$ \\
I got frustrated & 15 & $42.86 \%$ \\
I got bored & 5 & $14.3 \%$ \\
I was reprimand by others & 2 & $5.7 \%$ \\
\hline
\end{tabular}

Table 12: Reasons to uninstall the application. 35 women responded to this question and 5 were absent. Women could point out more than one reason, therefore the sum is not $100 \%$
When women were asked whether Tinder was developed by a man or a woman (question 10), $85.3 \%$ of the 34 who responded suggested that developer was a man (see table 13).

\begin{tabular}{lrr}
\hline Is the developer a Man or a Women? & No. of Women & $\%$ \\
\hline Man & 29 & $85.3 \%$ \\
Both & 4 & $11.8 \%$ \\
Woman & 1 & $2.9 \%$ \\
\hline Total respondents & 34 & $100 \%$ \\
\hline
\end{tabular}

Table 13: Developer's gender according to the interviewees. 34 women responded to the question, four had no opinion and one was absent.

When asked if there is any perceived difference in men's and women's motivations in using Tinder, $40 \%$ said there is no difference and $60 \%$ that they generally have different interests. Among those that think there is a difference, $95.83 \%$ said that men look for casual sex, while $4.17 \%$ that men are open to many things (relationship, casual sex, friendship). From the same $60 \%$ that think there is a difference, $70.83 \%$ think that women in general look for relationship while $29.17 \%$ believe women are open to many things.

In response to question $1,60 \%$ of the sample expressed interest in friendship (among other interests), 40\% interested in relationship, but from this $40 \%$, only half expressed exclusive interest in a relationship. That is, from the sample of 40 women only $22.5 \%$ were using the app exclusively for finding a partner. Conversely, from the same 40 women, $42.5 \%$ indicated that women are, in general, looking for relationship. Further, not a single women said that women are in general looking for friends, when $60 \%$ said to be interested in friendship and/or meeting "new people" in question 1, which was the top-rated interest among them. From the original sample of 40 women, 39 were looking for heterosexual interaction. Cross-tabulation between responses to question 1 and question 11 (see table 14) shows that from people looking for heterosexual relationship and/or friends but not for casual sex ( 30 women), 20 women believe that men are generally looking for casual sex. Thus, $2 / 3$ of the women who are only looking for relationship and/or friendship believe that men are not, in general. They represent $50 \%$ of the total sample. They're are looking for relationship or friendship but rather they feel that men are using the application for different purposes than theirs.

Comparing the results of question 11 with the results for the bad experiences (question 7) and the results for the developer's gender (question 10) we see the correlation between the $70 \%$ of women who have experienced harassment, the $60 \%$ that think men and women generally have different interests in the application and the $85.3 \%$ that think the developer is a man may be a sign that the application is mostly developed to meet male's expectations. On the assumption that it is the case the application may incite gender imbalance and sexual harassment.

In response to question 13, the interviewees suggested improvements that would provide them a better experience. The most rated improvement is the addition of filters which is related to their previous answers about first impression and negative side of the application. They have reported that a filter regarding the purpose of use would save time, avoid asymmetry of interest, bring confidence and 


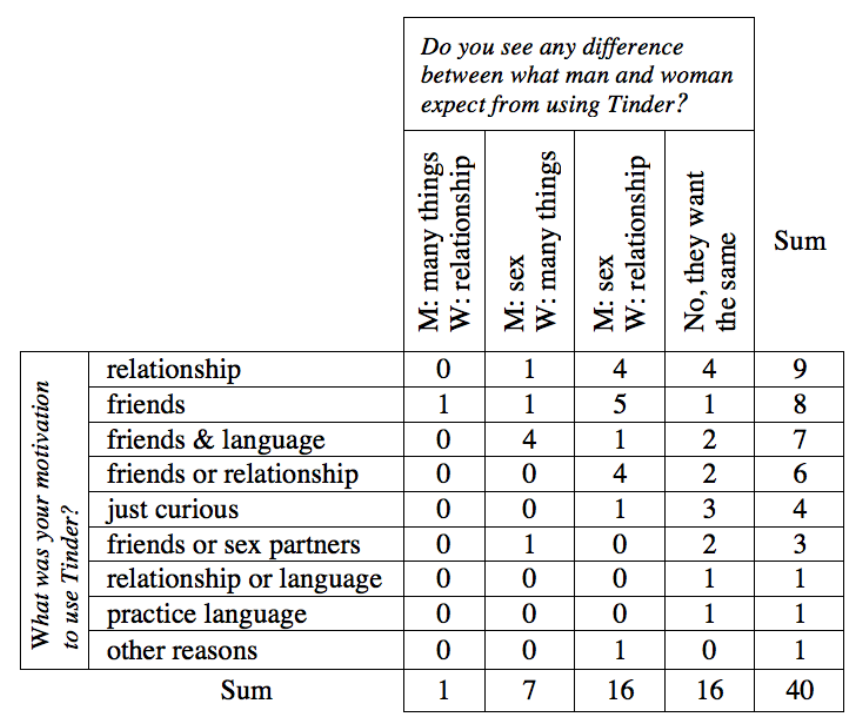

Table 14: Comparison between women's motivations on Tinder and women's perception of difference between men's and women's motivations.

increase the feeling of safety during the interaction. They would also report concerns about some physical features like height that they could not estimate through pictures and that seems crucial for some of them. Some other filters like "visited places" would help to find people with shared interests. One important fact that emerges from responses to this question is that some women blame themselves for their bad experience, for example when they say that the experience would be better if they had fewer expectations, less hesitation or more patience.

When asked about other applications that the respondents have used for the same purpose, more than half of the sample who answered $(51.6 \%)$ have also used Happn and $35.5 \%$ have used POF. The results also show that $35.5 \%$ of the respondents would prefer to use Tinder rather than other dating applications, and Tinder is the most rated as a favorite among them. However, the sum of other applications prefered by them shows that $48.4 \%$ would prefer other application rather than Tinder and $16.1 \%$ did not express a preference but would identify negative and positive aspects in all those used by them.

\section{THE INTERACTION AND USER INTERFACE ANALYSIS}

Computer-mediated communication (CMC) relies on the design of the interaction and on the GUI to communicate the purpose of a interactive system. However, those systems contain, apart from explicit meanings, implicit meanings which speaks through design qualities [?]. The aesthetics of design comprises the complex symbolic representations [15] that represents social structures and beliefs.

Because our society has been developed to "afford" gender equality, users' perceptions regarding the interaction reveals not only their feelings but also the applications' affordances [9], which encompasses the design qualities [?]. We understand that the gender dynamic established on Tinder is encouraged by its graphical user interface and interaction design.

In this study we analyse the Tinder interface to seek understanding of the connection between users' feedbacks and the application GUI. However, we believe that to provide a more positive experience on online dating applications it is necessary to design a entirely new interaction from the beginning, involving users and professionals to promote pleasant experience for both male and female users. A redesign would help to mitigate some problems pointed here but it would not provide a substantial social change in online dating realm.

Tinder provides simple and fast means to engage people through mobile devices. Its innovation is underpinned by a simple interaction basically composed of two steps. On one hand, it requires time to view the profiles one by one, and this diminishes the effectiveness (but not the efficiency) of the application since users could reach more people (more effective, less time required) if they were all displayed in a scrollable grid view or list view instead (see figure 2) as used by Happn, for example. On the other hand, it may enable more matches since one does not know what comes next and users might tend to consider more what they have in view. Further, considering the concept of classical conditioning [16] where the body learns through repetition how to be rewarded, the matches would represent a positive reinforcer that would incite users to keep swiping to get more rewards. Tinder's graphical user interface also connotes a deck of cards, and that contributes to why it seems fun and playful for some users. However, for others, it can exacerbate the superficiality of the interface: choosing and discarding people instead of cards.
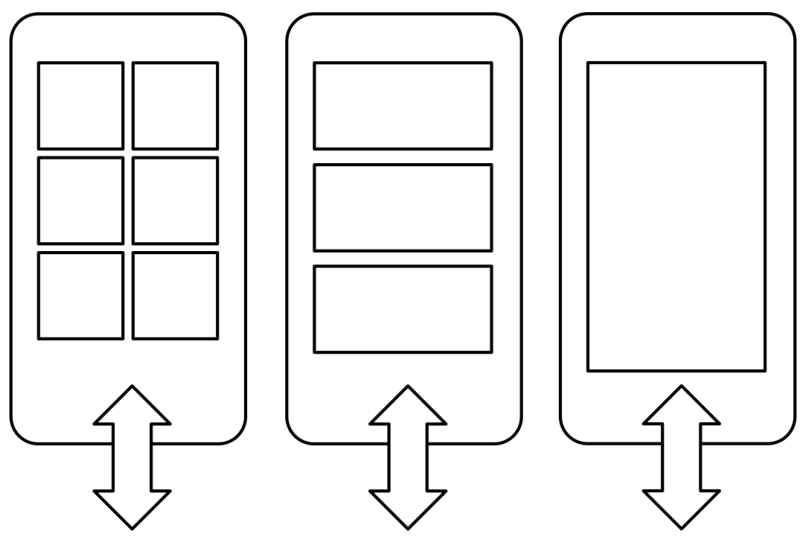

Figure 2: Scrollable grid and list view options for displaying information on the screen.

Regarding the efficacy, Tinder has proven to be efficacious in achieving users' goals, especially in relation to making friends and finding partners for long-term-relationships, which are the most indicated interests. Concerning the experience on Tinder, the majority $(52.6 \%)$ expressed having a negative first impression of the application. The application match system is considered superficial by a relevant number of users in this research $(37,5 \%)$, nonetheless it enhances privacy and reciprocity. Privacy and reciprocity in turn 
enhances confidence, as discussed in the opening sections of this paper.

Probably the superficiality of the Tinder experience does not inhere in the match system but in the whole interface which could be deemed excessively simple and based on appearances only. The "disposability of people" and the superficiality of a system based on appearance bring to light the concept of liquid love proposed by Bauman [3]. The liquidity of relationships refers to the ease of connecting to and disconnecting from people that internet make possible. There are so many contacts number in the list, it is so easy to connect making a call or texting, that the fragility of those connections is not a matter. The boundless network and the uncountable possibilities built up a new crowd of self-propelled people who do not fear losing connections [3]. With the abundant availability of connections it is simple to bond with people and even simpler to break up with them. The design of a new interaction focused on the positive act of "choosing" instead of "picking or discarding" would also reduce the superficiality and maintain the "positive rewards" for the match system.

The lack of filters is one of the recurrent complaints related to superficiality which could be easily solved with more filter options, but especially with a filter of "interest" that would bring honesty and clarity to the user's purposes. The superficiality might be diminished if users could set their purposes, common visited places, tastes, and so on. For some people, even diet counts because it is for them a matter of conscience. Many users will define it in their profiles, but there is no way to filter by "vegan" or "vegetarian", or by places that users like to go. The female users also said that some physical attributes as height is decisive and should be explicit in the profiles and possibly used as a filter, however, besides worsening the superficiality, it would be at odds with the promotion of diversity, it would incite the excluding standards of beauty which is also one of the aggravating factors of the objectification of women.

All these improvements of the profile and the possibility to filter by interests would enhance users to make use of their "personal front" which was first defined as the mix of appearance and manner that people use to present themselves to others [10]. However, through the virtual space the personal front would be mostly defined by appearance and interests since Tinder still don't have any audio or video features to support the "presentation of the self". Possibly new features would come up with this opportunities in mind.

In relation to "concerns" prior to installation, the results show that the majority feel free to use an online dating application and were not worried about consequences. It is a good sign that women are interested in technological approaches to relationships, and there is a fertile field to explore regarding technological developments and online dating. Some women are concerned about being recognized or are skeptical about this kind of approach, however they will still install it because they believe it is worth a try. It could be possible to develop some kind of invisibility to a company, a college or a neighbourhood, however it would reinforce the fear and the idea of hiding sexuality as something to be ashamed of. Online dating applications can be an extension of the sexual revolution, and this positive use of internet is possible when there is a mutual respect and equalitarian rights are emphasized.
Apart from the "like-dislike" perspective, Tinder's interaction has some useful features to enhance self-esteem. The match system enhances privacy and reciprocity because one obtains only positive feedback from the application: Tinder will indicate when two people reciprocally like each other. Furthermore, usually one will not remember their "likes" because there is no record of the people one has liked. Users can only keep track of the people they have matched. That feature avoid the feeling of rejection and positively emphasizes the successful numbers: the amount of matches. Additionally, the super like feature is also a boost of self-esteem considering that those who give it may nott keep track of it but it will possibly make a positive difference to those who receive it.

Analysing the application and interview results, it is possible to make a link between the interface design and the sexist behavior evidently experienced by participants in the study reported here. $70 \%$ of all the interviewees have reported offensive behavior regarding gender dynamics and pointed it out as the worse side of their experience. As social dynamics speaks through behavior as well as through language [5], this number givess a brief idea of how the sexist thought is still determining social relations and reinforcing the gender patterns that threaten women's empowerment and gender balance within labor, family and community realms.

Many of the expectations projected over women in relation to being "vulnerable" are set up during the childhood ${ }^{2}$ when "little innocent girls" may be "in danger" and should be protected from sexual abuse [19]. However, how is the idea of "vulnerability" treated in their adult lives, when they are "unprotected" by their families? In both moments women are taken as an object of an uncontrolled male sexuality, which traces are still active behind the norms of behavior, as shown by the results of this research. They are even considered dangerous due to the potential sex crimes they could incite [19]. This dynamic of predator-unsafety prey is exacerbated in online dating applications due to the perception of internet as a "free-zone" (virtual social context), where people feel more free to say what they would not dare to say in other circumstances (and real social contexts). That is the main concern about the application that inspired this research. Considering contemporary gender dynamics, it could be very harmful to women. It threatens their confidence, blocks their empowerment process, reinforces the idea of women as sexual objects and makes room for abusive/offensive behavior.

Some positive approaches to the interaction could make online dating applications also a space to spread equality through the concept of mutual respect. One possibility is that users would receive a good rating and be more visible when they develop a respectful behavior and that they would depend at least partly on the others' feedback to gain visibility (and possibly also in part on automatic classification of their within-system texts in relation to abusive or respectful language [11]). In association with the "positive reward" of increased visibility, users would be encouraged to develop good manners during their interaction with Tinder. However, their good manner would depend not only on the other user feedback, but also on a common effort towards mutuality. That is, good ratings for behavior would be possible if both users decide to help each other and only when both are committed to the rating system. If the rating could be visible only to the user rated it would provide feedback

${ }^{2}$ See, for example, "Little Red Riding Hood". 
about his/her behavior and an indication that his or her profile would or not be boosted as a reward. As an alternative to ratings, it could also be measured by the duration of the interaction or by the exchange of personal information (Facebook page, telephone number, email), and so on. Apart from the positive outcome of the promotion of good behavior and rewards within the virtual space it is very likely that it would also enhance good behavior in the reality as a consequence of the classical conditioning inherent in the suggestion.

As a result of the improvement of behavior, female users would probably feel more respected during their experience, since the majority we interviewed did not feel entirely respected, which should be a fundamental goal of online dating application. The highly rated reasons for uninstalling for frustration show that it is very likely that the application does not meet women's expectations and needs. Motivations comes from a natural desire to feel pleasure and avoid pain but to engage people in the activity is necessary to sustain the pleasure [6]. Pleasure in a broad sense refers to the social, emotional, physical or intellectual pleasure. Even if a relationship urge or a sex drive are the initial motivators, it is necessary to improve users satisfaction on different levels to engage them to Tinder.

Furthermore, the answers regarding the developer's gender show that women may feel a male dominance in technological development or that the application was developed to meet men's interests and expectations. However, even if they think that it was developed by male only because there are more men developing applications (due to gender imbalance in education), the predominance of male in technological development will consequently imply a development driven by male interests.

Regarding other online dating applications, Tinder was the best rated among them, however the majority still prefer to use other applications but Tinder for the purpose. Happn was the second most rated and according to this research would be the most relevant competitor of Tinder. However, it is the result of a small sample. The main differences from Tinder are: the grid view already explained above, the proximity by GPS (since the users can only access people that they have crossed path with), and a counter for the number of times the user crosses path with those people.

The results of this study reveal that Tinder is not meeting women's expectations and needs in relation to the application performance and that it has been a space to the replication of sexist patterns and the reinforcement of superficiality. Yet, it is a useful tool to connect them to friends and partners. To make mobile environments also a space for women's empowerment it is necessary to involve women in the development of the interaction and interface, as users, as thinkers, and as developers.

\section{CHALLENGES AND CONSTRAINTS}

This first study based on interviews intended to provide an overview of women's experience of Tinder in relation to gender dynamics in order to inform the design of a subsequent survey with a more quantitative approach. From the categorization enabled by the qualitative interviews and the descriptive statistics generated, it will be possible to developed a focused questionnaire to carry on quantitative research. Structured user tests may also enable to go deeper into the psychological aspects of the interaction and women's expectations to bring out ideas on how to empower women in a very sensitive matter as establishing relationships through mobile applications is. Because engagement today is still full of sexist patterns both in real and virtual environment, it is urgent to bring solutions that transform the virtual environment into a source of empowerment and equality.

Given this potentially gender-sensitive tentative conclusion, it must be considered the extent to which this might generalize beyond the sample involved as participants in the study, to all women. Some standard potential critiques of the work must therefore be addressed at this point, most importantly: that there might be sampling bias, that the linguistic and cultural context may have influenced response, that the conduct of the study might have induced demand characteristics [17]. It must be acknowledged that the sample is limited, and future work would gain by involving more participants in follow-up studies. This, however, does not invalidate the conclusions reached here, as while they clearly do not generalize to all women (none of the responses were unanimous), if the conclusions are appropriate to a group of $N 40$ women, then they nearly certainly are appropriate to $N 1$ (and so on).

As interview analysis is the first step to a deeper study on gender dynamics within mobile applications, the challenge here was to bring out the expressions of gendered power relationship in the virtual social interactions and to carefully transform the raw reports into categorised data that could be statistically handled and analysed later. The whole process required many reviews of the raw data and the statistics. Only through repeated close readings we could avoid bias during the process, since the intrinsic psychological analysis of the interviews that enabled to transform the paragraphs of answers into words is sensitive.

\section{CONCLUSIONS}

The initial interpretation of results of the interviews presented here suggests that Tinder has provided a space to reinforce a gender imbalance and that Tinder does not meet women's needs and expectations regarding online dating. On the other hand, Tinder has proved to be highly effective in bringing people together and promoting interactions.

Whilst we cannot reduce relationships to a binary gendering perspective, the study of male-female dynamic is still very important for the understanding of the hidden beliefs of gender hierarchy which promotes a gender imbalance. That dynamic is not only seen within heterosexual and homosexual interactions but also within familiar structure, labor structures, circles of friends, among others. The patterns of sexism impinge on all of society's layers. Virtual environments could offer a means to re-educate society in actual environments.

To provide females a positive experience on Tinder a deep understanding of well-being must be considered. Well-being depends on factors like self-awareness, self-esteem and empathy to promote joy through different aspects of human-computer engagement (cognitive, behavioral, emotional and agentic) [6]. A close look at how the gender imbalance is reflected on women's experiences and well-being will give clues to improving design interactions, and these may help to mitigate misogyny and sexist behaviors.

Finally, but no less importantly, we do not ignore the urgency of involving male users in future research and to promote the development of equality in consonance with a broader sense of respect 
which includes all the users and their motivations, regardless of any personal dissonance or evolutionary differences (where they exist).

\section{ACKNOWLEDGEMENTS}

The researchers have no conflicts of interest with Tinder nor any comparable platform. The first author wishes to acknowledge the Brazilian government's CAPES program.

This research is supported by Science Foundation Ireland (SFI) through the CNGL Programme (Grant 12/CE/I2267 and 13/RC/2106) in the ADAPT Centre (www.adaptcentre.ie).

\section{A STRUCTURED INTERVIEW QUESTIONS}

1) Why did you install the application? What were you looking for and what were your expectations?

2) Did you have to ponder before installing it? Why?

3) When you started using it, what was your first impression? How did you feel about your first matches?

4) How did you feel about the application?s approach and the matchbased interaction?

5) Which are the positive aspects of your experience? Tell me about the some remarkable situations.

6) Which are the negative aspects of your experience? Did anything unpleasant happen? Tell me about some situations.

7) Did you feel respected as an individual and as a woman? Explain. 8) For how long have you been using/ have used the application?

9) If you have stop using the application, what is the reason?

10) Do you think the developer was a man or a woman?

11) Do you see any difference between what man and woman expect from using the application? What are they looking for, in general?

12) Do you have female friends that are using / have used the application? What did you heard from them?

13) How could your experience be more pleasant?

14) Apart from Tinder, have you used another relationship application? Which one do you prefer?

\section{B INTERVIEW QUESTIONS SPLIT FOR ANALYSIS}

1) Why did you install the application? What were you looking for and what were your expectations?

2) Did you have to ponder before installing it? Why?

3) When you started using it, what was your first impression?

3-X)How did you feel about your first matches?

4) How did you feel about the application?s approach and the matchbased interaction?

5) Which are the positive aspects of your experience? Tell me about the some remarkable situations.

6) Which are the negative aspects of your experience? Did anything unpleasant happen? Tell me about some situations.

7) Did you feel respected as an individual and as a woman? Explain.

8) For how long have you been using/ have used the application?

9) Did you stop using the application?

9-X) What is the reason for unistalling?

10) Do you think the developer was a man or a woman?

11) Do you see any difference between what man and woman expect from using the application? What are they looking for, in general?
12) Do you have female friends that are using / have used the application? What did you heard from them?

13) How could your experience be more pleasant?

14) Apart from Tinder, have you used another relationship application?

14-X)Which one do you prefer?

\section{REFERENCES}

[1] 2016. (November 2016). https://www.gotinder.com/press

[2] 2017. Advances in the Understanding of Same-Sex and Opposite Sex Sexual Harassment. Evolution and Human Behavior (2017)

[3] Zygmunt Bauman. 2003. Liquid Love: on the frailty of human bonds. Polity Press, Cambridge, UK.

[4] Melanie Birks and Jane Mills. 2015. Grounded Theory: A practical Guide. SAGE.

[5] Lisa Blackman. 2008. The Body: The Key Concepts. Berg Publishers, Oxford, UK.

[6] Rafael A. Calvo and Dorian Peters. 2014. Positive Computing: Technology for Wellbeing and Human Potential. The MIT Press.

[7] Juliet Corbin and Anselm Strauss. 2008. Basics of Qualitative Research: Techniques and Procedures for developing Grounded Theory. SAGE.

[8] John W. Creswell. 2014. Research Design: Qualitative, Quantitative, and Mixed Methods Approach. SAGE.

[9] James J Gibson. 1979. The ecological approach to visual perception. Houghton Mifflin, Boston, MA.

[10] Erving Goffman. 1959. The Presentation of Self in Everyday Life. Penguin Books, London, UK.

[11] Van Hee, Cynthia, Els Lefever, Ben Verhoeven, Julie Mennes, Bart Desmet, Guy De Pauw, Walter Daelemans, and Véronique Hoste. 2015. Automatic detection and prevention of cyberbullying. In International Conference on Human and Social Analytics (HUSO 2015). IARIA, 13-18.

[12] Claire Hewson, Carl Vogel, and Dianna Laurent. 2016. Internet Research Methods, 2nd Ed. London: SAGE.

[13] Jie Liang Lin. 2017. Digital Environments: Ethnographic Perspectives Across Global Online and Offline Spaces. Transcript Media Studies, Chapter Antifeminism Online: MGTOW (Men Going Their Own Way), 77-96.

[14] Michael Quinn Patton. 2002. Qualitative Research and Evaluation Methods. SAGE.

[15] Marianne Graves Petersen, Ole Sejer Iversen, Peter Gall Krogh, and Martin Ludvigsen. 2004. Aesthetic Interaction: a Pragmatist's Aesthetics of Interactive Systems. In 5th conference on Designing interactive systems: processes, practices, methods, and techniques (DIS '04).

[16] Edmund T. Rolls. 2007. Emotion Explained. Oxford University Press, New York, NY.

[17] Robert Rosenthal. 1967. Covert communication in the psychological experiment. Psychological Bulletin 67 (1967), 356-367.

[18] Judy Wajcman. 2004. Technofeminism. Polity Press, Cambridge, UK.

[19] Valerie Walkerdine. 1997. Daddy's Girl: Young Girls and Popular Culture. MacMillan Press LTD, London, UK. 